\title{
HIDROCARBONETOS POLICÍCLICOS AROMÁTICOS COMO TRAÇADORES DA QUEIMA DE CANA-DE- AÇÚCAR: UMA ABORDAGEM ESTATÍSTICA
}

Dulce Magalhães

Centro de Química e Meio Ambiente, Instituto de Pesquisas Energéticas e Nucleares, 05508-000 São Paulo - SP, Brasil

Roy E. Bruns

Instituto de Química, Universidade de Campinas, 13084-862 Campinas - SP, Brasil

Pérola de Castro Vasconcellos*

Instituto de Química, Universidade de São Paulo, CP 26077, 05513-970 São Paulo - SP, Brasil

Recebido em 17/2/06; aceito em 17/7/06; publicado na web em 10/1/07

\begin{abstract}
POLYCYCLIC AROMATIC HYDROCARBONS AS SUGARCANE BURNING TRACERS: A STATISTICAL APPROACH. In this study atmospheric particulates of PAHs were measured in Araraquara, Piracicaba and São Paulo in July 2003 (sugarcane harvest season in Araraquara and Piracicaba) and in Araraquara in March of 2003. The results were normalized to the total PAH concentrations. Comparison among the sites and principal component analysis (PCA) were used to investigate possible tracers of emission. Fluoranthene and pyrene concentrations were higher in Piracicaba and Araraquara samples. These PAH were also responsible for the largest negative loadings on the second principal component and account for the negative scores and for the formation of the Araraquara and Piracicaba group.
\end{abstract}

Keywords: PAH; sugarcane burning; PCA.

\section{INTRODUÇÃO}

Embora grande produtor de açúcar desde o final do século XVI, o Brasil expandiu muito a cultura da cana-de-açúcar a partir da década de 70, com o advento do PROÁLCOOL, programa desenvolvido pelo governo brasileiro para estimular a produção de etanol, usado como combustível de automóveis. Atualmente, o Brasil possui 4,5 milhões de ha de terra plantados com cana-de-açúcar. A produção brasileira vem aumentando a cada ano. Na safra de 2003/04 foram produzidas 359 milhões de t de cana. O Brasil é o maior produtor de cana-deaçúcar do mundo, seguido por Índia e Austrália. A cana é colhida no Centro-Sul (de maio a outubro) e no Norte-Nordeste (dezembro a maio), o que permite dois períodos de safra. Na média, 55\% da cana brasileira gera álcool e $45 \%$ açúcar ${ }^{1-3}$.

O Estado de São Paulo possui 2,35 milhões de ha de terra plantados com cana de açúcar e responde por $60 \%$ da produção nacional. São Paulo é também responsável por $64 \%$ do álcool e $56 \%$ do açúcar produzidos no Brasil ${ }^{3}$.

Na época da safra, as plantações de cana-de-açúcar são queimadas. A cana passa por uma queima pré-colheita para facilitar o trabalho dos cortadores e protegê-los, pois elimina do local o excesso de folhas e palhiço e espanta os animais peçonhentos (cobras, escorpiões etc.) muito comuns nas plantações. A queima da cana também melhora o rendimento do corte manual (aumentado em até 10 vezes), auxilia no preparo do terreno para novos plantios e aumenta a quantidade de açúcar por peso, devido à evaporação da água ${ }^{1,2}$.

A prática de queimar palha de cana-de-açúcar acarreta problemas de poluição do ar em razão da grande emissão de fumaça e fuligem que podem atingir os centros urbanos, trazendo sérios transtornos à população das cidades canavieiras. A fuligem, que permanece por longo tempo em suspensão no ar, é considerada uma das causas da alta incidência de doenças respiratórias no Brasil ${ }^{1,4}$.

*e-mail: perola@iq.usp.br
As queimadas são consideradas a principal fonte de emissão de material particulado (MP) no mundo. Estima-se que sua contribuição mundial esteja na ordem de $104 \mathrm{Tg} / \mathrm{ano}^{1}$. No Brasil, as queimadas de cana-de-açúcar contribuem sem dúvida com emissões de material particulado ${ }^{1,5}$, no entanto, mais estudos devem ser realizados para avaliar esta contribuição. Além disso, são necessárias mais informações em relação à composição química desta fuligem e ao possível perigo que possa causar à saúde humana e ao meio ambiente ${ }^{1,5,6}$.

A queima da biomassa introduz diversos compostos na atmosfera, incluindo compostos carcinogênicos e/ou mutagênicos, como os hidrocarbonetos policíclicos aromáticos (HPA) ${ }^{1,4,7}$.

Os HPA caracterizam-se por possuírem dois ou mais anéis aromáticos condensados. São formados durante a combustão incompleta ou pirólise de materiais contendo carbono e hidrogênio e estão presentes na atmosfera tanto na fase gasosa como na particulada. A contribuição de fontes biogênicas de HPA é limitada, restringindo-se praticamente à queima espontânea de florestas e emissões vulcânicas. As fontes antrópicas representam o principal processo de emissão. As queimadas, a queima de combustíveis como petróleo e seus derivados, carvão, madeira, gás de carvão, fumaça de cigarro e calefação (especialmente em países de clima temperado) são importantes fontes de $\mathrm{HPA}^{8-10}$.

Segundo a literatura, os HPA podem ser usados como indicadores de fontes de emissão. O perfil dos HPA ou a abundância relativa de HPA individuais em emissões de diversas fontes de combustão são sugeridos como assinaturas confiáveis de fontes onde marcadores inorgânicos não estão disponíveis ${ }^{11-13}$.

O uso dos HPA como traçadores de queima de florestas já foi relatado na literatura ${ }^{14,15}$.

No presente estudo foram analisados os HPA presentes no MP das amostras coletadas no Estado de São Paulo nas cidades de Araraquara (ARA), Piracicaba (PIR) e São Paulo (SPA) em julho de 2003 (período de safra da cana-de-açúcar em ARA e PIR) e Araraquara (ARAe) em março de 2003 (época de entressafra).

Os níveis dos HPA no material particulado foram sujeitos à aná- 
lise de componentes principais para estudar os fatores que contribuem para a maior parte da variação química. Os gráficos bidimensionais das componentes principais permitem a visualização desta variação e, por isto, contém mais informação estatística que os gráficos envolvendo somente as medidas originais. No caso da existência de agrupamentos de pontos nestes gráficos, as medidas químicas que têm as maiores diferenças entre os grupos podem ser determinadas. Se estes agrupamentos podem ser relacionados com os diferentes locais de amostragem, podemos identificar as substâncias químicas que são mais características de cada local de amostragem. Sendo um procedimento multivariado, os componentes principais permitem um estudo das correlações entre as medidas químicas e, desta maneira, complementam os métodos univariados que normalmente ficam restritos ao cálculo das médias e desvios padrão de cada variável em cada agrupamento das amostras.

\section{PARTE EXPERIMENTAL}

\section{Sítios de amostragem}

A Figura 1 mostra os sítios de amostragem no Estado de São Paulo.

A cidade de Araraquara localiza-se a $282 \mathrm{~km}$ da capital do estado, com cerca de 182.000 habitantes (censo 2000). A sua estrutura industrial está baseada na agroindústria, representada pelo binômio cana e laranja. Outros setores de destaque da economia local são o setor mecânico, metalúrgico, têxtil, de produtos alimentares e de vestuário. Araraquara está situada no centro do assim chamado "cinturão da cana-de-açúcar". É considerada uma área modelo para estudos da degradação ambiental causada pela prática das queimadas de cana-de-açúcar. Nesta região, há poucas indústrias e não existem cidades vizinhas de grande porte ${ }^{4,16,17}$.

Piracicaba está localizada a $175 \mathrm{~km}$ da capital do Estado e possui cerca de 300.500 habitantes. Situa-se em uma das regiões mais industrializadas do estado. O seu complexo industrial é formado por mais de 5000 indústrias, destacando-se entre as variadas atividades, os setores metalúrgico, siderúrgico, mecânico, têxtil, alimentício e petroquímico. No setor agrícola destacam-se as culturas de cana-de-açúcar (10 milhões de t por ano), café (1 milhão de pés) e laranja (6 milhões de pés) ${ }^{18}$.

A região metropolitana de São Paulo (RMSP), com uma área aproximada de $8.000 \mathrm{~km}^{2}$, tem uma população superior a 17 milhões de habitantes, distribuída de modo desordenado em uma área urbanizada de $1747 \mathrm{~km}^{2}$. É o terceiro maior conglomerado urbano do mundo. A região sofre todo tipo de problemas ambientais, entre os quais está a deterioração da qualidade do ar, devido às emissões atmosféricas de cerca de 2000 indústrias de alto potencial poluidor e uma frota aproximada de 7,8 milhões de veículos (20\% do total nacional). O sítio de amostragem está localizado na região oeste da cidade de São Paulo, no Campus da Universidade de São Paulo, uma área arborizada que está sujeita a um intenso tráfico de veículos dentro e fora do

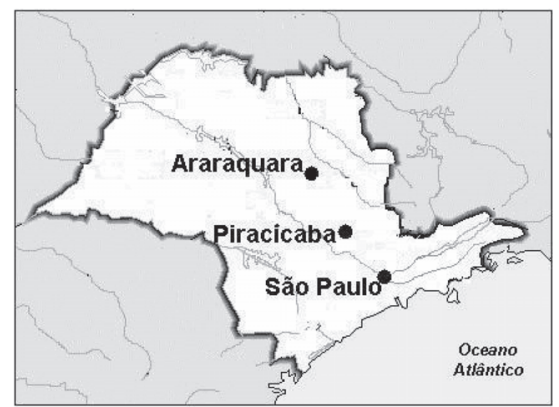

Figura 1. Sítios de amostragem
Campus. Além disso, o Campus está situado próximo à cidade de Osasco, cujo parque industrial engloba principalmente indústrias metalúrgicas, têxteis, alimentícias e de madeiras ${ }^{19,20}$.

\section{Amostragem}

As amostragens foram realizadas em amostradores de grande volume $\left(\mathrm{Hi}\right.$ - $\mathrm{Vol}$ ) com fluxo de $1,13 \mathrm{~m}^{3} / \mathrm{min}$ durante $24 \mathrm{~h}$. Nos sítios de São Paulo (15 amostras), Araraquara na safra (15 amostras) e na entressafra (10 amostras) foram coletadas partículas inaláveis menores que $10 \mu \mathrm{m}\left(\mathrm{MP}_{10}\right)$ e no sítio de Piracicaba (14 amostras) foi coletado o material particulado total (MPT) para partículas $<100 \mu \mathrm{m}$.

Em todos os sítios foram utilizados filtros de fibra de quartzo (20x25 cm), exceto em Araraquara (amostragem de março de 2003), onde foram usados filtros de teflon. Todos os filtros de fibra de quartzo foram previamente aquecidos em mufla a $800{ }^{\circ} \mathrm{C}$ durante 8 h para remoção de impurezas orgânicas. Após a amostragem, os filtros foram estocados a $-10{ }^{\circ} \mathrm{C}$.

\section{Determinação dos HPA}

O material orgânico particulado foi extraído dos filtros com 200 $\mathrm{mL}$ de diclorometano em aparelho de Soxhlet durante $24 \mathrm{~h}$ (60 ciclos). Após a extração, o solvente foi evaporado em rotavapor à 40 ${ }^{\circ} \mathrm{C}$ e o extrato concentrado sob fluxo de $\mathrm{N}_{2}$ à temperatura ambiente.

Os extratos das amostras foram separados por cromatografia à líquido de alta eficiência (CLAE) em fase normal usando n-hexano e diclorometano em gradiente de concentração. O volume injetado foi $20 \mu \mathrm{L}$ em uma vazão de $1 \mathrm{~mL} / \mathrm{min}$. A fração de HPA foi obtida através de uma coluna Nucleosil 100-10 (25 cm x 4,6 mm x 10 $\mu \mathrm{m})$ em cromatógrafo Shimadzu equipado com detector UV-Vis modelo SPD-10AV ajustado em $254 \mathrm{~nm}$. O volume da fração coletada foi reduzido em fluxo de $\mathrm{N}_{2}$ e as frações foram analisadas usando-se cromatógrafo a gás Shimadzu modelo GC-17 equipado com detector de ionização de chama (DIC) e uma coluna capilar DB-5 (30 m x 0,25 mm x 0,25 $\mu \mathrm{m})$. Todas as injeções foram "splitless" e o volume injetado foi igual a $1 \mu \mathrm{L}$. As temperaturas usadas no detector e injetor foram, respectivamente, 320 e $300{ }^{\circ} \mathrm{C}$. O programa da temperatura do forno variou de $100{ }^{\circ} \mathrm{C}$ (por $1 \mathrm{~min}$ ) a $280{ }^{\circ} \mathrm{C}$ (por 26,5 min). A taxa de aquecimento utilizada foi de 8 ${ }^{\circ} \mathrm{C} \min ^{-1}$.

A identificação dos HPA nas amostras foi feita por comparação com os tempos de retenção de uma mistura padrão (16 HPA) do fornecedor Supelco (USA). Na quantificação foi usado o método do padrão externo. As curvas analíticas foram preparadas a partir de 6 soluções com concentrações diferentes da mistura padrão. $\mathrm{Na}$ quantificação do Benzo(e)pireno foi usada a curva do Benzo(a)pireno. O detector por ionização de chama é um detector massa-sensitivo, que responde ao número de átomos de carbono que entram no detector por unidade de tempo. Assim sendo, os compostos estudados B(a)P e B(e)P apresentam o mesmo fator de resposta.

Os coeficientes de correlação $\left(\mathrm{R}^{2}\right)$ obtidos das curvas foram maiores que 0,9980 e os limites de detecção variaram de 0,25 a 1,65 ng.

Nos testes de recuperação foi utilizada a mistura padrão de HPA do fabricante Supelco e o padrão certificado NIST. O material de referência padrão NIST - SRM 1649 refere-se a "Material Particulado de Poeira Urbana”. Os testes de recuperação foram feitos através da adição de uma quantidade conhecida da mistura padrão em um filtro, o qual foi, então, submetido ao mesmo processo de extração e separação utilizado nas amostras. A eficiência de recuperação com a mistura padrão de HPA variou de 58,3 a 92,2\% (média 81,3\%) e com o padrão NIST variou de 72,8 a 106,2\% (média 91,3\%).

Neste estudo foram analisados 13 HPA, 12 dos quais estão en- 
tre os 16 HPA incluídos na lista de poluentes prioritários da Agência de Proteção Ambiental dos Estados Unidos (EPA), mais o Benzo(e)pireno, citado pelo Instituto Nacional de Saúde Ocupacional e Segurança (NIOSH) ${ }^{21}$.

Os HPA de acordo com sua ordem de eluição foram: Fenantreno (Fen), Antraceno (Ant), Fluoranteno (Flu), Pireno (Pir), Benzo(a)antraceno (BaA), Criseno (Cri), Benzo(b)fluoranteno (BbF), Benzo(k)fluoranteno (BkF), Benzo(e)pireno (BeP), Benzo(a)pireno (BaP), Indeno(1,2,3-cd)pireno (Ind), Dibenzo(a,h)antraceno (DBA) e Benzo(ghi)perileno (BPe).

\section{Análise de componentes principais}

A análise de componentes principais (PCA) é uma técnica estatística multivariada usada para reduzir o dimensionamento dos dados e formar um conjunto menor de fatores ortogonais de mais fácil interpretação. O princípio desta análise é transformar o conjunto original de variáveis em um conjunto menor de combinações lineares, que responda pela maior parte da variância do conjunto original. A função primária desta análise é a redução do número de variáveis mantendo a informação original tanto quanto possível, de modo que as variáveis com características semelhantes possam ser agrupadas em fatores ${ }^{11,22}$.

\section{RESULTADOS E DISCUSSÃO}

\section{Concentrações relativas dos HPA}

A Tabela 1 mostra as concentrações relativas (\%) dos HPA nos quatro sítios analisados. Para efeito de comparação entre os sítios, as concentrações foram normalizadas em relação à soma do 13 HPA identificados.

Tabela 1. Concentrações em \% dos HPA individuais

\begin{tabular}{lcccc}
\hline & SPA & ARA & PIR & ARAe \\
HPA & $\%$ & $\%$ & $\%$ & $\%$ \\
\hline Fen & 1,2 & 3,2 & 2,0 & 1,4 \\
Ant & 1,3 & nd & nd & 5,2 \\
Flu & 2,3 & 8,3 & 6,3 & 2,5 \\
Pir & 2,5 & 9,3 & 6,8 & 2,6 \\
BaA & 16,1 & 11,5 & 15,4 & 7,8 \\
Cri & 9,7 & 8,7 & 8,2 & 10,3 \\
BbF & 5,6 & 5,9 & 6,1 & 2,6 \\
BkF & 6,8 & 5,7 & 4,8 & 2,0 \\
BeP & 11,9 & 11,6 & 12,3 & 11,5 \\
BaP & 11,3 & 8,4 & 10,4 & 7,8 \\
Ind & 13,8 & 12,6 & 12,9 & 2,3 \\
DBA & 4,3 & 0,2 & 1,9 & 0,7 \\
BPe & 12,9 & 14,7 & 12,9 & 43,3 \\
S HPA & 100,0 & 100,0 & 100,0 & 100,0 \\
\hline As concen
\end{tabular}

As concentrações foram normalizadas em relação à soma dos 13 HPA identificados.

Pela Tabela 1 pode-se observar que os compostos dominantes em São Paulo são BaA (16\%), Ind (14\%), BPe (13\%), BeP (12\%) e $\mathrm{BaP}(11 \%)$. Os outros 8 compostos representam $34 \%$ da concentração total de HPA neste sítio. Em Piracicaba, os principais HPA são $\mathrm{BaA}(15 \%)$, Ind (13\%), BPe (13\%), BeP (12\%) e $\mathrm{BaP}(10 \%)$. Os outros HPA somam $37 \%$.

Em São Paulo e Piracicaba os HPA mais abundantes foram os mesmos, o que sugere que existem fontes em comum nesses dois sítios. Alguns estudos sugerem que $\mathrm{BaP}, \mathrm{BaA}$ e $\mathrm{BeP}$ se originam em grande quantidade de produtos do aço ${ }^{11}$.

Em Araraquara (safra), os compostos mais abundantes são BPe (15\%), Ind (13\%), BeP (12\%), BaA (12\%) e Pir (9\%). Os outros HPA totalizam $39 \%$.

Enquanto isso, em Araraquara na entressafra, BPe (43\%) é o HPA mais abundante. BPe representa quase a metade dos HPA identificados nesse sítio. Segundo a literatura ${ }^{23,24}$, BPe pode ser usado como traçador de emissões veiculares, o que sugere a importância das fontes veiculares em Araraquara no mês de março. Os demais HPA identificados em Araraquara (entressafra) são BeP (11\%), Cri (10\%), BaP (8\%), BaA (8\%) e outros $(20 \%)$.

A concentração relativa dos HPA (Tabela1) Fen, Flu e Pir é mais alta em Araraquara (safra) (3,2; 8,3 e 9,3\%) e Piracicaba (2,0; $6,3$ e $6,8 \%)$ que em São Paulo $(1,2 ; 2,3$ e $2,5 \%)$ e Araraquara na entressafra $(1,4 ; 2,5$ e $2,6 \%)$. Ant não foi detectado em Araraquara e Piracicaba. De acordo com a literatura ${ }^{15}$, os principais HPA emitidos pela queima das gramíneas são: Fen, Flu e Pir com pequena contribuição de Ant. Estudos anteriores citam Pir como traçador de queima da biomassa ${ }^{14}$.

A Figura 2 mostra as concentrações relativas de Fen, Flu, Pir e Ant nos sítios de São Paulo, Araraquara, Piracicaba e Araraquara (entressafra).

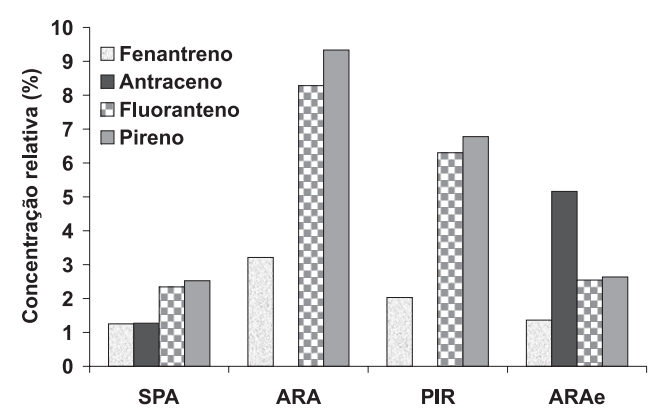

Figura 2. Comparação dos HPA (Fen, Ant, Flu e Pir) entre os sítios

A Figura 2 mostra um perfil semelhante em Araraquara (safra) e Piracicaba, o que sugere a contribuição das queimadas de canade-açúcar que ocorrem em ambos os sítios no mês de julho.

\section{Análise dos componentes principais (PCA)}

A análise dos componentes principais, efetuada através do programa Statistica versão $6.0^{25}$, foi feita a partir de dados normalizados. Foi formado um conjunto de dados com 13 variáveis e 54 amostras, o que forneceu um total de 702 observações. Foram retidos os componentes principais com autovalores $>1$.

A PCA indicou que 74,4\% da variância total dos dados são explicados por quatro componentes principais. A PCA1 e a PCA2 representam $52,1 \%$ da variância total. A Tabela 2 mostra os pesos dos fatores mais importantes para cada componente principal.

O fator 1 (PC1) explicou 31,5\% da variância dos dados. Os HPA dividiram-se em dois grupos. Um deles é formado por Fen, Flu, Pir, $\mathrm{BaA}, \mathrm{BbF}, \mathrm{BkF}, \mathrm{BeP}, \mathrm{BaP}$, Ind e DBA com pesos negativos e o outro por Ant e BPe com pesos positivos. No primeiro grupo, $\mathrm{BbF}$ e $\mathrm{BkF}$ apresentaram pesos altos enquanto que Ind teve peso moderado. BbF, BkF e Ind são considerados bons marcadores de emissões de veículos a diesel ${ }^{22,26}$. Alguns autores consideram o Ind um traçador de combustão de diesel ${ }^{26}$. No segundo grupo, Ant e BPe mostraram pesos altos. Estudos citam o BPe como um HPA característico de emissões de veículos a gasolina ${ }^{27}$. Outros trabalhos associam Ant e BPe a resíduos de óleo ${ }^{26}$. No presente estudo, o PC1 foi associado a emissões veiculares (diesel e gasolina) e resíduos de óleo. 
Tabela 2. Pesos dos fatores extraídos dos componentes principais

\begin{tabular}{lcccc}
\hline & 1 & 2 & 3 & 4 \\
\hline Fen & $-0,346$ & $-0,348$ & 0,577 & - \\
Ant & $\mathbf{0 , 7 7 5}$ & - & - & - \\
Flu & $-0,392$ & $\mathbf{- 0 , 8 6 7}$ & - & - \\
Pir & $-0,489$ & $\mathbf{- 0 , 8 4 0}$ & - & - \\
BaA & $-0,387$ & - & $-0,385$ & 0,558 \\
Cri & - & 0,360 & 0,332 & 0,672 \\
BbF & $\mathbf{- 0 , 8 0 1}$ & - & - & - \\
BkF & $\mathbf{- 0 , 7 5 2}$ & - & $-0,316$ & - \\
BeP & $-0,394$ & - & 0,581 & - \\
BaP & $-0,475$ & 0,382 & 0,543 & $-0,410$ \\
Ind & $-0,511$ & - & $-0,517$ & - \\
DBA & $-0,327$ & $\mathbf{0 , 7 9 6}$ & - & - \\
BPe & $\mathbf{0 , 9 3 3}$ & - & - & - \\
\hline
\end{tabular}

Valores com módulos menores que 0,250 foram omitidos; valores com módulos maiores que 0,700 estão em negrito

O fator 2 (PC2) explicou 20,6\% da variância total. Os HPA neste fator também se dividiram em dois grupos. Um deles é formado por Fen, Flu e Pir com pesos negativos e o outro por DBA, Cri e BaP com pesos positivos. No primeiro grupo, Flu e Pir tiveram pesos altos ao passo que Fen teve peso baixo. Fen, Flu e Pir são considerados os principais HPA emitidos pela queima das gramíneas ${ }^{15}$ e Pir é citado como traçador de queima da biomassa ${ }^{14}$. Nesse estudo, os HPA Fen, Flu e Pir foram associados às queimadas de cana-de-açúcar. O segundo grupo apresenta DBA com peso alto e Cri e $\mathrm{BaP}$ com pesos baixos. Cri, BaP e DBA estão relacionados a emissões de fontes estacionárias $^{11}$. DBA está também relacionado com usinas termelétricas que usam carvão como combustível ${ }^{11}$. O PC2 foi associado às queimadas de cana-de-açúcar e às fontes estacionárias (indústrias).

O fator 3 (PC3) explicou 12,7\% da variância total. Neste fator nenhum HPA apresentou peso alto. Fen, BeP, BaP e Cri formam um grupo com pesos positivos, no qual Fen, BeP e BaP apresentam pesos médios e Cri peso baixo. Fen, $\mathrm{BeP}$ e $\mathrm{BaP}$ já foram encontrados em combustão de madeira ${ }^{12}$. Alguns estudos mostram que BaP pode ser um bom traçador para combustão de madeira ${ }^{13}$. O outro grupo é formado por Ind, $\mathrm{BaA}$ e $\mathrm{BkF}$ com pesos negativos. Ind teve peso médio ao passo que $\mathrm{BaA}$ e $\mathrm{BkF}$ tiveram pesos baixos. Devido às baixas correlações entre esses HPA não foi possível associar este grupo a nenhuma fonte específica. $\mathrm{O}$ fator 3 foi associado à combustão de madeira.

O fator 4 (PC4) explicou 9,5\% da variância dos dados. Neste fator encontra-se um grupo com BaA e Cri com pesos positivos (médios) e outro com BaP com peso negativo (baixo). Alguns estudos mostram que BaA e Cri estão associados à combustão de gás natural e consideram $\mathrm{BaA}$ um traçador da combustão de gás natural ${ }^{28}$. O PC4 foi associado à combustão deste gás.

A Figura 3 mostra os escores do Fator 1 e Fator 2 extraídos da análise de componentes principais.

Os escores podem ser descritos pelas seguintes equações aproximadas

Fator $1=+0,775[$ Ant $]-0,801[\mathrm{BbF}]-0,752[\mathrm{BkF}]+0,933[\mathrm{BPe}]$

onde [Ant], [BbF], [BkF] e [BPe] são as concentrações normalizadas e autoescalonadas de Ant, BbF, BkF e BPe.

Fator $2=-0,348[$ Fen $]-0,867[$ Flu $]-0,840[$ Pir $]+0,360[$ Cri $]+$ $0,382[\mathrm{BaP}]+0,796[\mathrm{DBA}]$

onde [Fen], [Flu], [Pir] [Cri], [BaP] e [DBA] são as concentrações

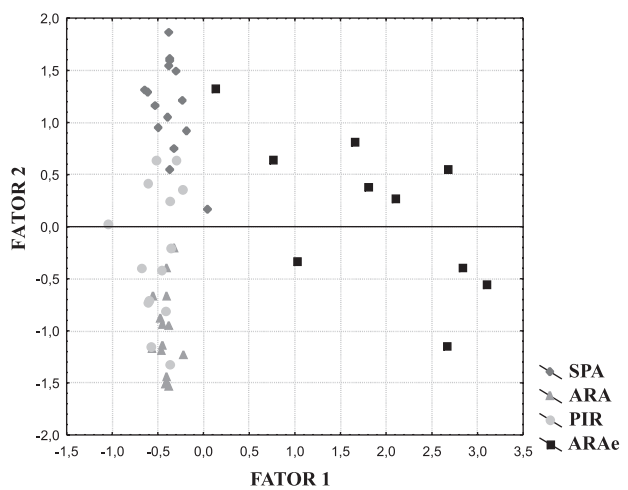

Figura 3. Escores extraídos da análise de PCA

normalizadas e autoescalonadas de Fen, Flu, Pir Cri, BaP e DBA.

Pela análise dos escores extraídos da análise de componentes principais, São Paulo, Piracicaba, Araraquara e Araraquara (entressafra) formam quatro grupos distintos, indicando que os HPA presentes nesses sítios se originam de fontes diferentes. As amostras de Piracicaba distribuem-se entre o conjunto formado pelas amostras de Araraquara e São Paulo, sugerindo que Piracicaba tem fontes de HPA em comum com esses dois sítios. A maioria das amostras de Piracicaba está junto com as de Araraquara (safra), o que indica que os HPA presentes em Piracicaba e Araraquara têm mais fontes em comum. Além disso, as amostras de Araraquara e Araraquara (entressafra) encontram-se em grupos distintos, apesar de pertencerem ao mesmo sítio, indicando que as fontes de HPA que estão atuando nos meses de julho e março são de origens diferentes.

O primeiro componente principal discrimina as amostras de Araraquara (entressafra) das de São Paulo, Araraquara (safra) e Piracicaba. Os escores positivos na PC1 das amostras de Araraquara (entressafra) resultam de concentrações relativamente altas de Ant e de BPe, que têm pesos positivos neste fator, e concentrações relativamente baixas de $\mathrm{BbF}$ e $\mathrm{BkF}$, que têm pesos negativos. Por outro lado, as amostras de São Paulo, Araraquara (safra) e Piracicaba têm escores negativos na $\mathrm{PC} 1$ porque têm concentrações mais altas de $\mathrm{BbF}$ e $\mathrm{BkF}$ que as amostras de Araraquara (entressafra). Isto pode ser verificado na Tabela 1, especialmente para BPe com 43,3\% que já foi destacado neste trabalho.

O segundo componente principal distingue as amostras de São Paulo da maioria das amostras de Piracicaba e Araraquara (safra). As concentrações relativas de todos os HPA (Tabela 1), exceto Flu e Pir, foram mais altas nas amostras de São Paulo que nas de Araraquara (safra) e Piracicaba. Flu e Pir tiveram os pesos negativos maiores no PC2 e são responsáveis pelos escores negativos e pela formação de um grupo com amostras de Araraquara e Piracicaba no lado esquerdo inferior. Em contraste, as concentrações relativas de DBA foram muito mais altas nas amostras de São Paulo que nas de Araraquara e Piracicaba, o que explica os escores positivos e a formação de um grupo com amostras de São Paulo no lado esquerdo superior. Embora existam poucas amostras de Piracicaba com escores positivos, elas estão posicionadas entre as amostras de São Paulo e Araraquara (safra).

\section{CONCLUSÃO}

Os HPA mais abundantes em São Paulo e Piracicaba foram $\mathrm{BaA}$, Ind, $\mathrm{BPe}, \mathrm{BeP}$ e BaP, indicando possíveis fontes comuns nesses sítios. Em Araraquara os principais HPA encontrados foram $\mathrm{BPe}$, Ind, BeP, BaA e Pir e em Araraquara (entressafra) foram BPe, BeP, Cri, BaP e BaA.

As concentrações relativas de Fen, Flu e Pir, compostos 
traçadores de queima de gramíneas foram mais altas em Araraquara (safra) e Piracicaba que em São Paulo e Araraquara (entressafra).

A análise dos componentes principais mostrou que os HPA fluoranteno e pireno foram responsáveis pela discriminação das amostras de Araraquara (safra) e a maioria das amostras de Piracicaba, das amostras de São Paulo. Estas substâncias têm os maiores pesos negativos no PC2 e este componente pode ser associado com queima da cana-de-açúcar (gramíneas). Por outro lado, o PC1 aparentemente representa as emissões veiculares e de queima dos resíduos de óleo.

As observações acima sugerem a influência das queimadas de cana-de-açúcar nas emissões de Araraquara e Piracicaba durante o período de safra, sendo os compostos fluoranteno e pireno, os marcadores dessas emissões.

As fontes de emissão identificadas neste estudo resultaram de informações que constam na literatura e da análise de componentes principais do conjunto de dados. Elas não foram obtidas diretamente das fontes emissoras.

\section{AGRADECIMENTOS}

Ao LEMA (Laboratório de Estudos do Meio Ambiente) do IQUSP pelo uso do laboratório; à FAPESP, Fundação de Amparo à Pesquisa do Estado de São Paulo, pelo suporte financeiro (Projeto 2001/0176-3).

\section{REFERÊNCIAS}

1. Santos, C. Y. M.; Azevedo, D. A.; Neto, F. R. A.; Atmos. Environ. 2002, $36,3009$.

2. http://www.cori.rei.unicamp.br, acessada em Agosto 2004.
3. http://www.unica.com.br, acessada em Abril 2005.

4. http://www.cetesb.sp.gov.br/ar/relatorios/relatorios.asp, acessada em Maio 2004.

5. Allen, A. G.; Cardoso, A. A.; Rocha, G. O.; Atmos. Environ. 2004, 38, 5025.

6. http://www.usp.br/agenciausp, acessada em Janeiro 2006.

7. Kalaitzoglou, M.; Terzi, E.; Samara, C.; Atmos. Environ. 2004, 38, 2545.

8. Lopes, W. A.; de Andrade, J. B.; Quim. Nova 1996, 19, 497.

9. Vasconcellos, P.; Tese de Doutorado, Universidade de São Paulo, Brasil, 1996.

10. Vasconcellos, P.; Artaxo, P. E.; Ciccioli, P.; Cecinato, A.; Bracaleoni, E.; Frattoni, M.; Quim. Nova 1998, 21, 385.

11. Fang, G. C.; Chang, C. N.; Wu, Y. S.; Fu, P. P. C.; Yang, I. L.; Chen, M. H.; Sci. Total Environ. 2004, 327, 135.

12. Khalili, N. R.; Scheff, P. A.; Holsen, T. M.; Atmos. Environ. 1995, $29,533$.

13. Kulkarni, P.; Venkataraman, C.; Atmos. Environ. 2000, 34, 2785.

14. Masclet, P.; Cachier, H.; Liousse, C.; Wortham, H.; J. Atmos. Chem. 1995, $22,41$.

15. Simoneit, B. R. T.; Appl. Geochem., 2002, 17, 129.

16. Godoi, R. H. M.; Godoi, A. F. L.; Worobiee, A.; Andrade, S. J.; Hoog, J.; Santiago-Silva, M. R.; Grieken, R.V.; Microchim. Acta 2004, 145, 53.

17. http://www.araraquara.sp.gov.br. acessada em Maio 2005.

18. http://www.ciagri.usp.br. acessada em Maio 2005.

19. http://www.cetesb.sp.gov.br/ar/relatorios/relatorios.asp, acessada em Outubro 2005.

20. http://www.citybrazil.com.br. acessada em Maio 2005.

21. Zamperlini, G. C. M.; Silva, M. S.; Vilegas, W. R.; J. Chromatogr., A 2000, 889, 281.

22. De Luca, G.; Furesi, A.; Leardi, R.; Micera, G.; Panzanelli, G.; Piu, P. C.; Sanna, G.; Mar. Chem. 2004, 8, 15.

23. Harrison, R. M.; Smith, D. J. T.; Luhana, L.; Environ. Sci. Technol. 1996, 30,825 .

24. Miguel, A. H.; Pereira, P. A. P.; Aerosol Sci. Technol. 1989, 10, 292.

25. Statsoft, Inc.; Statistics 6.0, 2300 E $14^{\text {th }}$ St. Tulsa, OK, USA.

26. Kavouras, I. G.; Koutrakis, P.; Tsapakis, M.; Lagoudaki, E.; Stephanou, E. G.; Von Baer, D.; Oyola, P.; Environ. Sci. Technol. 2001, 35, 2288.

27. Bourotte, C.; Forti, M. C.; Taniguchi, S.; Bícego, M. C.; Lotufo, P. A.; Atmos. Environ. 2005, 39, 3799.

28. Simcik, M. F.; Eisenreich, S. J.; Lioy, P. J.; Atmos. Environ. 1999, 33, 5071. 